\title{
Comparison of Sirolimus and Paclitaxel-Eluting Stents for Complex Coronary Lesions: An Intravascular Ultrasound Study
}

\author{
Yun-Kyeong Cho, Seung-Ho Hur, Hyun-Tae Kim, In-Cheol Kim, Hyoung-Seob Park, Hyuck-Jun Yoon, Chang-Wook \\ Nam, Hyungseop Kim, Seong-Wook Han, Yoon-Nyun Kim, and Kwon-Bae Kim
}

Division of Cardiology, Department of Internal Medicine, Dongsan Medical Center, Keimyung University College of Medicine, Daegu, Korea

Background/Aims: Recent intravascular ultrasound (IVUS) studies of sirolimus-eluting stents (SES) and paclitaxeleluting stents (PES) have demonstrated a significant reduction in neointimal hyperplasia (NIH) based on simple coronary lesions. In this study, we evaluated the efficacy of SES and PES using IVUS in complex coronary lesions. Methods: Eighty-seven patients in whom 95 drug-eluting stents (66 SES and 29 PES) were implanted in complex coronary lesions were enrolled in this study. Case selection was based on the availability of IVUS and quantitative coronary angiographic (QCA) examinations at the index procedure and at follow-up. The neointimal volume index (volume/length: NIVI) and percent neointimal volume (\% NIV) were calculated. The longitudinal length of stented segments without IVUS-detectable NIH was also evaluated.

Results: The baseline patient demographics were similar between the SES and PES groups. At follow-up, no significant differences were observed in the vessel, plaque, or stent volume indices between the two groups. However, the NIVI and \% NIV were significantly lower in the SES group $(p<0.01)$. The longitudinal length of stented segments without IVUS-detectable NIH was significantly higher in the SES group $(p<0.01)$. The net gain was significantly larger in the SES group $(2.3 \pm 0.7$ vs. $2.0 \times 0.6 \mathrm{~mm}, p=0.025)$, while the rate of major adverse cardiac events was similar between the two groups.

Conclusions: Although SES showed significantly greater suppression of $\mathrm{NIH}$ at follow-up, both stents were highly effective at inhibiting NIH in complex coronary lesions. (Korean J Intern Med 2009;24:323-329)

Keywords: Coronary artery disease; Drug-eluting stents; Ultrasonography

\section{INTRODUCTION}

The introduction of drug-eluting stents (DES) led to a significant decrease in angiographic and clinical restenosis by inhibiting smooth muscle cell migration and proliferation compared with bare-metal stents [1-4]. By demonstrating differences in the rate of target lesion revascularization and major adverse cardiac events (MACE), most studies confirmed the superiority of DES. In addition, a number of studies indicated that both sirolimus-eluting stents (SES) and paclitaxel-eluting stents (PES) significantly decreased the rate of angiographic restenosis despite differences in late loss [3-6]. However, these studies mostly focused on simple coronary lesions, although DES tend to be used in patients with more complex lesions, which are associated with higher rates of MACE. In addition, in the evaluation of procedural results, two-dimensional coronary angiography provides limited information about lesion characteristics, while intravascular ultrasound (IVUS) provides unique and more accurate information regarding neointimal hyperplasia (NIH), vessel remodeling, and stent apposition [2]. Although a few studies have evaluated the

Received: January 25, 2009

Accepted: June 3, 2009

Correspondence to Seung-Ho Hur, M.D.

Division of Cardiology, Department of Internal Medicine, Dongsan Medical Center, Keimyung University College of Medicine, 194 Dongsan-dong, Jung-gu, Daegu 700-712, Korea

Tel: 82-53-250-7411, Fax: 82-53-250-7034, E-mail: shur@dsmc.or.kr 
Table 1. Baseline clinical and angiographic characteristics

\begin{tabular}{|c|c|c|c|}
\hline & $\begin{array}{c}\text { SES } \\
(n=62)\end{array}$ & $\begin{array}{c}\text { PES } \\
(n=29)\end{array}$ & $p$ value \\
\hline Age, yr & $56.9 \pm 9.1$ & $58.7 \pm 10.4$ & NS \\
\hline Male & 74.4 & 82.1 & NS \\
\hline Diabetes mellitus & 20.9 & 17.9 & NS \\
\hline Hypertension & 37.2 & 42.9 & NS \\
\hline Hyperlipidemia & 18.6 & 10.7 & NS \\
\hline Smoking & 48.8 & 28.6 & NS \\
\hline Previous MI & 2.3 & 7.1 & NS \\
\hline Previous $\mathrm{PCl}$ & 2.3 & 28.6 & 0.002 \\
\hline LVEF & $55.1 \pm 11.8$ & $52.4 \pm 10.2$ & NS \\
\hline ACS & 67.4 & 64.3 & NS \\
\hline Multi-vessel disease & 30.2 & 28.6 & NS \\
\hline Target vessel & & & 0.004 \\
\hline Left anterior descending artery & 72.1 & 46.4 & \\
\hline Left circumflex artery & 0 & 21.4 & \\
\hline Right coronary artery & 27.9 & 32.1 & \\
\hline Reference vessel diameter, $\mathrm{mm}$ & $3.3 \pm 0.3$ & $3.3 \pm 0.5$ & NS \\
\hline Lesion length, mm & $23.5 \pm 9.0$ & $24.4 \pm 9.4$ & NS \\
\hline Pre-minimal luminal diameter, $\mathrm{mm}$ & $0.5 \pm 0.3$ & $0.5 \pm 0.4$ & NS \\
\hline Diameter stenosis & $84.7 \pm 9.4$ & $84.9 \pm 11.8$ & NS \\
\hline Lesion type & & & NS \\
\hline A & 0 & 0 & \\
\hline B1 & 14.0 & 14.3 & \\
\hline B2 & 18.6 & 21.4 & \\
\hline $\mathrm{C}$ & 67.4 & 64.3 & \\
\hline Ostial lesion & 12.9 & 24.1 & NS \\
\hline Bifurcation lesion & 14.5 & 20.7 & NS \\
\hline Left main lesion & 8.1 & 13.8 & NS \\
\hline Chronic total occlusion & 12.9 & 13.8 & NS \\
\hline Stent size, $\mathrm{mm}$ & $3.3 \pm 0.3$ & $3.3 \pm 0.5$ & NS \\
\hline Stent length, mm & $27.0 \pm 10.1$ & $26.4 \pm 9.0$ & NS \\
\hline Maximal inflation pressure, atm & $16.4 \pm 2.1$ & $14.9 \pm 2.2$ & 0.004 \\
\hline Anti-platelet therapy & & & NS \\
\hline Aspirin/clopidogrel & 39.5 & 57.1 & \\
\hline Aspirin/clopidogrel/cilostazol & 60.5 & 42.9 & \\
\hline
\end{tabular}

Values are presented as mean \pm SD or percentages.

SES, sirolimus-eluting stents; PES, paclitaxel-eluting stents; NS, no significance; MI, myocardial infarction; PCl, percutaneous coronary intervention; LVEF, left ventricular ejection fraction; ACS, acute coronary syndrome.

efficacy of DES based on IVUS parameters, IVUS findings using a 40-MHz transducer with high-quality image acquisition for complex lesions have not been fully elucidated. Therefore, the present study was performed to evaluate and compare NIH between DES in complex lesions using IVUS with a 40-MHz transducer.

\section{METHODS}

\section{Study population and protocol}

Eighty-seven patients who had undergone successful stent implantation with 95 DES and post-stenting IVUS were enrolled in this study. The SES group consisted of 59 patients with 62 native coronary lesions (66 DES), while the PES group consisted of 28 patients with 29 native coronary lesions (29 DES). Signed informed written consent was obtained from all patients prior to the study.

Patients with signs or symptoms of ischemia who met the criteria for complex patient characteristics or complex lesions as follows were enrolled in this study: diabetes, acute coronary syndrome, lesion types B2 and C according to the American Heart Association, bifurcation lesions, recent and chronic total occlusions, ostial lesions, lesions 
Table 2. Angiographic and clinical results

\begin{tabular}{|c|c|c|c|}
\hline & $\begin{array}{c}\text { SES } \\
(n=62)\end{array}$ & $\begin{array}{c}\text { PES } \\
(n=29)\end{array}$ & $p$ value \\
\hline \multicolumn{4}{|l|}{ Post-procedure } \\
\hline Final MLD, mm & $3.0 \pm 0.3$ & $3.0 \pm 0.4$ & NS \\
\hline Diameter stenosis & $8.8 \pm 3.1$ & $9.3 \pm 3.9$ & NS \\
\hline \multicolumn{4}{|l|}{ Follow-up } \\
\hline Final MLD, mm & $2.78 \pm 0.5$ & $2.5 \pm 0.7$ & 0.026 \\
\hline Diameter stenosis & $15.1 \pm 13.8$ & $21.5 \pm 15.9$ & NS \\
\hline Acute gain, $\mathrm{mm}$ & $2.6 \pm 0.4$ & $2.5 \pm 0.5$ & NS \\
\hline Late loss, mm & $0.2 \pm 0.5$ & $0.5 \pm 0.6$ & 0.034 \\
\hline Net gain, mm & $2.3 \pm 0.7$ & $2.0 \pm 0.6$ & 0.025 \\
\hline MACE & 1.6 & 6.9 & NS \\
\hline Death & 0 & 0 & NS \\
\hline $\mathrm{Ml}$ & 0 & 0 & NS \\
\hline TLR & 1.6 & 3.4 & NS \\
\hline TVR & 1.6 & 6.9 & NS \\
\hline Stent thrombosis & 0 & 3.4 & NS \\
\hline Binary restenosis & 0 & 6.9 & NS \\
\hline
\end{tabular}

Values are presented as mean \pm SD or percentages.

SES, sirolimus-eluting stents; PES, paclitaxel-eluting stents; MLD, minimal lumen diameter; NS, no significance; MACE, major adverse cardiac events; MI, myocardial infarction; TLR, target lesion revascularization; TVR, target vessel revascularization.

in the proximal left anterior descending artery, long lesions ( $>16 \mathrm{~mm}$ ), or left main stem lesions. All patients were older than 18 years and underwent successful stent implantation with Thrombolysis in Myocardial Infarction (TIMI) 3 flow. IVUS examination was also performed after stent deployment at the index procedure, and the subjects underwent an eight-month IVUS examination as well as follow-up coronary angiography. Patients were excluded if they had: 1) intolerance or a contraindication to aspirin or clopidogrel, 2) advanced heart failure or an ejection fraction $<40 \%$, or 3 ) severe comorbidity.

All interventions were performed according to current standard guidelines. When preinterventional IVUS was performed, stent size was determined according to IVUS parameters. In cases without preinterventional IVUS, stent size was determined based on angiographic findings. When postdilatation was required to optimize stent expansion or apposition, a balloon shorter than the stent length was used with careful positioning of the balloon inside the stent to avoid injury at the edge. Cases in which incomplete stent apposition (ISA) was not resolved due to a large proximal reference vessel were classified as acute ISA. A final IVUS examination was performed after the last balloon inflation. The patients were premedicated with aspirin, which was continued indefinitely, and given clopidogrel (loading dose of $300 \mathrm{mg}$ ), which was initiated 24 hours before intervention. The patients were advised to stay on clopidogrel for $\geq 6$ months after stent deployment.

\section{Quantitative coronary angiographic (QCA) analysis}

The results of coronary angiography, which was performed according to standard techniques, were analyzed using a computer-assisted system for QCA analysis (Digital Cardiac Imaging System; Philips Medical Systems, Best, The Netherlands) using end-diastolic frames and a contrast-filled guiding catheter for calibration. The \% diameter stenosis was defined as [1(minimal lumen diameter/reference vessel diameter)] $\times 100$. Angiographic restenosis was defined as $>50 \%$ diameter stenosis at follow-up. The immediate enlargement of the luminal diameter produced by stent implantation (acute gain) and the subsequent reduction in luminal diameter from the time of intervention to follow-up (late loss) were calculated. The net gain was thus the sum of the offsetting effects of acute gain and late loss.

\section{Follow-up evaluation}

The baseline clinical characteristics, procedural characteristics, and clinical outcomes used in this study were obtained from electronic medical records. Follow-up angiography was performed after eight to twelve months with a clinical follow-up of twelve months following stent implantation. During the follow-up period, MACE, including 
Table 3. Serial three-dimensional ultrasound results

\begin{tabular}{|c|c|c|c|c|c|c|c|c|}
\hline & \multicolumn{3}{|c|}{$\begin{array}{l}\text { SES } \\
(n=62)\end{array}$} & \multicolumn{3}{|c|}{$\begin{array}{c}\text { PES } \\
(n=29)\end{array}$} & \multirow[t]{2}{*}{$p$ value $^{\dagger}$} & \multirow[t]{2}{*}{$p$ value $^{\ddagger}$} \\
\hline & PS & FU & $p$ value* & PS & FU & $p$ value* & & \\
\hline \multicolumn{9}{|c|}{ Proximal reference, $\mathrm{mm}^{3} / \mathrm{mm}$} \\
\hline VVI & $17.1 \pm 5.7$ & $16.8 \pm 4.1$ & NS & $16.0 \pm 5.4$ & $16.6 \pm 5.4$ & NS & NS & NS \\
\hline LVI & $9.3 \pm 3.5$ & $9.3 \pm 3.1$ & NS & $8.9 \pm 3.3$ & $8.8 \pm 3.7$ & NS & NS & NS \\
\hline PVI & $7.9 \pm 3.0$ & $7.4 \pm 2.4$ & NS & $7.1 \pm 3.0$ & $7.8 \pm 3.0$ & NS & NS & NS \\
\hline \multicolumn{9}{|c|}{ Stented segment, $\mathrm{mm}^{3} / \mathrm{mm}$} \\
\hline VI & $15.8 \pm 3.3$ & $15.9 \pm 3.1$ & NS & $16.2 \pm 4.4$ & $16.2 \pm 4.3$ & NS & NS & NS \\
\hline LVI & $7.4 \pm 1.1$ & $7.3 \pm 1.3$ & NS & $8.3 \pm 2.3$ & $7.2 \pm 2.3$ & $<0.01$ & NS & NS \\
\hline PVI & $8.4 \pm 2.6$ & $8.3 \pm 2.1$ & NS & $7.9 \pm 2.5$ & $8.0 \pm 2.5$ & NS & NS & NS \\
\hline SVI & $7.4 \pm 1.1$ & $7.5 \pm 1.4$ & NS & $8.3 \pm 2.3$ & $8.2 \pm 2.3$ & NS & NS & NS \\
\hline NIVI & 0 & $0.2 \pm 0.4$ & 0.001 & 0 & $0.9 \pm 0.9$ & $<0.01$ & NS & $<0.01$ \\
\hline$\% \mathrm{NIV}$ & 0 & $2.8 \pm 4.3$ & $<0.01$ & 0 & $11.9 \pm 10.2$ & $<0.01$ & NS & $<0.01$ \\
\hline \multicolumn{9}{|c|}{ Distal reference, $\mathrm{mm}^{3} / \mathrm{mm}$} \\
\hline VI & $11.9 \pm 4.8$ & $12.6 \pm 4.3$ & NS & $10.8 \pm 4.9$ & $11.5 \pm 4.2$ & NS & NS & NS \\
\hline LVI & $7.0 \pm 2.5$ & $7.6 \pm 2.4$ & NS & $6.6 \pm 3.0$ & $6.8 \pm 2.5$ & NS & NS & NS \\
\hline PVI & $5.0 \pm 3.1$ & $5.0 \pm 2.7$ & NS & $4.1 \pm 2.4$ & $4.7 \pm 2.6$ & 0.006 & NS & NS \\
\hline
\end{tabular}

SES, sirolimus-eluting stents; PES, paclitaxel-eluting stents; PS, post procedure; FU, follow-up; VVI, vessel volume index; LVI, lumen volume index; PVI, plaque volume index; SVI, stent volume index; NIVI, neointimal volume index; \% NIV, percent neointimal volume; NS, no significance.

$* p$ value, post-procedure vs. follow-up.

${ }^{\dagger} p$ value, SES vs. PES at post-procedure.

$\ddagger p$ value, SES vs. PES at follow-up.

cardiac death, non-fatal myocardial infarction, and target lesion revascularization, were recorded and evaluated.

\section{IVUS imaging protocol}

IVUS examinations were performed immediately and eight to twelve months after stent implantation. All IVUS images were acquired using a commercially available ultrasound system (Cardiovascular Imaging System; Boston Scientific Corp., Natick, MA, USA). A singleelement mechanical transducer (2.5 or 2.9 Fr with a 40MHz IVUS catheter; Boston Scientific Corp.) was used. After the intracoronary administration of $200 \mu \mathrm{g}$ of nitroglycerin, the imaging catheter was advanced distal to the stent and withdrawn with an automated pullback device at $0.5 \mathrm{~mm} /$ second through the stent to the proximal vessel segment. The ultrasound images were recorded on a CD for off-line analysis. The IVUS images were analyzed using commercially available planimetry software (EchoPlaque; Indec Systems, Santa Clara, CA, USA) according to previously validated and published protocols [7]. The lumen, stent, and vessel contours were traced manually at 1.0-mm axial intervals for both the stented and reference vessel segments (5-mm segment proximal or distal to the stent). The plaque area was calculated by subtracting the lumen area from the vessel area. In the stented segments, the neointimal contours were also traced. Simpson's method was used to calculate the volumes, which were adjusted by the length. The lumen volume index (LVI), stent volume index, vessel volume index (VVI), plaque volume index (PVI), and neointimal volume index (NIVI) were determined and expressed as $\mathrm{mm}^{3} / \mathrm{mm}$. The percent neointimal volume (\% NIV) was

Table 4. The Incidence of incomplete stent apposition

\begin{tabular}{lcccc}
\hline & $\begin{array}{c}\text { SES } \\
(\mathrm{n}=62)\end{array}$ & $\begin{array}{c}\text { PES } \\
(\mathrm{n}=29)\end{array}$ & $\begin{array}{c}\text { Both } \\
(\mathrm{n}=91)\end{array}$ & $p$ value \\
\hline Post-procedure & $11(17.7)$ & $3(10.3)$ & $14(15.4)$ & $\mathrm{NS}$ \\
Follow-up & $18(29.0)$ & $4(13.8)$ & $22(24.2)$ & $\mathrm{NS}$ \\
$\quad$ Persistent ISA & $7(11.3)$ & $3(10.3)$ & $10(11.0)$ & $\mathrm{NS}$ \\
Resolved ISA & $4(6.5)$ & $0(0.0)$ & $4(4.4)$ & $\mathrm{NS}$ \\
Late-acquired ISA & $11(17.7)$ & $1(3.4)$ & $12(13.2)$ & NS \\
\hline
\end{tabular}

Values are number (\%).

SES, sirolimus-eluting stents; PES, paclitaxel-eluting stents; ISA, incomplete stent apposition. 
calculated as the ratio of the neointimal volume and stent volume $\times 100$. NIVI and \% NIV may be used to evaluate the mean amount of neointima regardless of stent diameter or length. To examine the distribution pattern of $\mathrm{NIH}$, the percentage of stent length free from IVUSdetectable NIH was calculated. The longitudinal length of stented segments without IVUS-detectable NIH was determined then divided by the total stented segments in each case. ISA was defined as one or more struts clearly separated from the vessel wall with evidence of blood behind the strut in a segment not associated with any side branches [8,9]. Resolved ISA was defined as that observed at baseline but not at follow-up. Persistent ISA was defined as ISA observed at both baseline and follow-up. Late-acquired ISA was defined as ISA observed only at follow-up [10].

\section{Statistical analysis}

Statistical analysis was performed using SPSS version 15.0 (SPSS Inc., Chicago, IL, USA). Continuous data are presented as the mean \pm standard deviation while categorical data are presented as the frequency. Continuous variables were compared by paired or unpaired Student's $t$-tests. Categorical variables were compared by $\chi^{2}$ and Fisher's exact tests. A $p<0.05$ was considered statistically significant.

\section{RESULTS}

\section{Patients and lesion characteristics}

A total of 87 patients with 95 drug-eluting stents (SES group, 59 patients with 66 stents vs. PES group, 28 patients with 29 stents) were enrolled in this study. The baseline demographic and angiographic data are shown in Table 1. The baseline characteristics were similar between the SES and PES groups. The frequency of diabetic patients was $20.9 \%$ in the SES group and $17.9 \%$ in the PES group ( $p>0.05)$.

\section{Angiographic and clinical results}

Our angiographic and clinical results are listed in Table 2. The mean time to follow-up angiography was 12.5 months. Acute gain was similar between the groups (2.6 $\pm 0.4 \mathrm{~mm}$ in the SES group vs. $2.5 \pm 0.5 \mathrm{~mm}$ in the PES group), while late loss was significantly smaller in the SES group than in the PES group (0.2 \pm 0.5 vs. $0.5 \pm 0.6$ $\mathrm{mm}, p=0.034$ ). Thus, net gain was significantly greater in the SES group ( $2.3 \pm 0.7$ vs. $2.0 \pm 0.6 \mathrm{~mm}$, respectively; $p=0.025)$. However, none of the parameters could independently predict net gain. Differences in the instent restenosis rates between the two groups were not statistically significant, and there was only one case of stent thrombosis in the PES group. After one year, there were no differences in the rate of MACE between the two groups.

\section{IVUS results}

Our serial IVUS data are shown in Table 3. There were no differences in the baseline IVUS characteristics between the two groups; however, the NIVI and \% NIV in the stented segment at follow-up were significantly lower for those lesions treated with SES compared to PES (0.2 \pm 0.4 vs. $0.9 \pm 0.9 \mathrm{~mm}^{3} / \mathrm{mm}, 2.8 \pm 4.3$ vs. $11.9 \pm 10.2 \%$, respectively; all $p<0.01$ ). The LVI at follow-up was significantly smaller than the postprocedure value in the PES group $\left(8.3 \pm 2.3 \mathrm{~mm}^{3} / \mathrm{mm}\right.$ postprocedure vs. $7.2 \pm 2.3$ $\mathrm{mm}^{3} / \mathrm{mm}$ at follow-up, $p<0.01$ ). There were no significant differences in any of the other parameters within the stented segments. In the proximal and distal reference segments, the LVI, PVI, and VVI were not significantly different between the two groups; however, the PVI in the distal reference segment of the PES group was increased at follow-up ( $4.1 \pm 2.4$ vs. $4.7 \pm 2.6 \mathrm{~mm}^{3} / \mathrm{mm}, p=0.006$ ).

The percentage of stent length free from IVUS-detectable NIH was significantly longer in the SES group than in the PES group, indicating that SES had focal NIH while PES had diffuse $\mathrm{NIH}$ ( $51.1 \pm 33.8$ vs. $37.1 \pm 27.5 \%, p=0.02$ ).

\section{ISA}

ISA was observed in fourteen cases postprocedure and 22 cases at follow-up. Resolved ISA was observed in four patients (6.5\%) in the SES group. Persistent ISA was observed in seven patients (11.3\%) in the SES group and three patients (10.3\%) in the PES group, while lateacquired ISA was seen in eleven patients $(17.7 \%)$ in the SES group and in one patient (3.4\%) in the PES group. However, there was no significant difference in the incidence rate of ISA between the two groups. ISA at the index procedure occurred at the proximal edge of the stent and was related to discrepancies between stent size and proximal reference vessel size. The location of lateacquired ISA was at the distal edge in four lesions (33.3\%, four SES) and within the body of the stent in eight lesions (66.7\%, seven SES and one PES). Our results indicate that the main cause of late-acquired ISA was focal positive remodeling and not a decrease in plaque volume. 


\section{DISCUSSION}

This study demonstrates the superiority of SES compared to PES in the suppression of neointimal proliferation, although both stents were associated with low rates of binary restenosis and MACE in patients with complex lesion characteristics [11-17]. Although late loss in our study appeared to be somewhat higher than that in the prospectively randomized trial comparing the safety and efficacy of sirolimus-eluting versus paclitaxel-eluting coronary stents (REALITY trial) (0.09 $\pm 0.43 \mathrm{~mm}$ in SES vs. $0.31 \pm 0.44 \mathrm{~mm}$ in PES, $p<0.001$ ) [18], which assessed the effects of both DES in a head-to-head comparison, the results were comparable with those in two other studies: the randomized study of sirolimus- vs. paclitaxel-eluting stents for coronary revascularization (SIRTAX study) and a study of paclitaxel- vs. sirolimus-eluting stents for prevention of restenosis in diabetic patients with coronary artery disease (ISAR-DIABETES trial) $[19,20]$. The difference in late loss might be related to differential inclusion criteria for the patients, in that patients with more complex characteristics were enrolled in this study compared to the REALITY study. There have been few comparative studies of the efficacy of SES and PES in reducing NIH by IVUS. The NIH volume in the present study was $5.8 \pm 12.3 \mathrm{~mm}^{3}$ in the SES group and $23.6 \pm 21.0$ $\mathrm{mm}^{3}$ in the PES group $(p<0.01)$. These values correspond to those from a previous study, which showed a significantly lower NIH volume in lesions treated with SES compared to PES. Cervinka et al. [21] reported that SES had a markedly reduced NIH volume compared to PES based on IVUS measurements $\left(4.1 \pm 11.0 \mathrm{~mm}^{3}\right.$ in SES vs. $17.4 \pm 23.0 \mathrm{~mm}^{3}$ in PES, $p=0.002$ ), however, the amount of NIH was evaluated based on the total volume regardless of stent length. In the present study, however, the degree of NIH was adjusted for the stent length by dividing the NIV by the stent length to remove any bias caused by differences in the length of each stent. Petronio et al. [22] also reported the degree of neointimal proliferation in DES. They included complex lesions in only the proximal and/or midportion of the left anterior descending artery and assessed neointimal proliferation as the NIH area (0.61士 $0.41 \mathrm{~mm}^{2}$ in SES vs. $1.20 \pm 0.56 \mathrm{~mm}^{2}$ in PES, $p<0.001$ ), their results are comparable to those of the present study.

To evaluate the lesions by IVUS, we used a transducer with a frequency of $40 \mathrm{MHz}$. Compared to the images provided by solid-state systems at $20 \mathrm{MHz}$, those obtained using a mechanical system at $40 \mathrm{MHz}$ are slightly better, which may enable a more precise evaluation of neointimal proliferation.

In most previous studies, determinations of the NIH pattern were made based on angiographic measurements. Using this method, it was widely reported that the NIH of the SES group was of the focal type while that of the PES group was predominantly of the diffuse type $[23,24]$. However, coronary angiography provides only indirect information about restenosis. We evaluated the distribution of NIH more quantitatively in our study through IVUS; the portion with or without NIH was validated while the proportion of stent length free from IVUS-detectable NIH was calculated as the number of NIH-free frames divided by the total number of frames. Among the various trials that have analyzed the efficacy of DES, this is one of the only studies to provide the NIH pattern through quantification by IVUS; our results show a similar NIH distribution to that reported by Petronio et al. [22] (69.8 \pm 29.4 vs. $43.9 \pm 34.8 \%, p<0.001)$.

The incidence rate of late-acquired ISA in the SES group was $17.7 \%$, which is similar to the results of previous studies [25,26]. On the other hand, in the PES group, the incidence rate of late-acquired ISA was $3.4 \%$, which is lower than the value reported previously [27]. The lower incidence in the PES group may be related to the small number of patients. There were no negative clinical events after twelve months, similar to other studies [10,26,27].

This study was limited by the small number of patients enrolled. The sample size was too small to compare the clinical events, but this was not an endpoint of the present study. In addition, this was a retrospective and nonrandomized study from a single center. Thus, selection bias cannot be excluded, and it likely affected the baseline characteristics. However, such differences had no influence on the outcomes. Finally, preinterventional IVUS was not performed in all patients, which may have affected the determination of the appropriate stent size at the time of the index procedure.

In summary, compared with PES, SES showed greater suppression of NIH at follow-up. However, both SES and PES were highly effective at inhibiting NIH in patients with complex characteristics, and there were no significant differences in clinical outcome among the limited number of cases studied during the relatively short follow-up period. Additional, large-scale prospective randomized trials with longer-term IVUS follow-up are needed to confirm these findings. 


\section{Acknowledgements}

This work was supported by a grant (No. RTIo4-O1o1) from the Regional Technology Innovation Program of the Ministry of Knowledge Economy (MKE).

\section{REFERENCES}

1. Morice MC, Serruys PW, Sousa JE, et al. A randomized comparison of a sirolimus-eluting stent with a standard stent for coronary revascularization. N Engl J Med 2002;346:1773-1780.

2. Moses JW, Leon MB, Popma JJ, et al. Sirolimus-eluting stents versus standard stents in patients with stenosis in a native coronary artery. N Engl J Med 2003;349:1315-1323.

3. Colombo A, Drzewiecki J, Banning A, et al. Randomized study to assess the effectiveness of slow- and moderate-release polymerbased paclitaxel-eluting stents for coronary artery lesions. Circulation 2003;108:788-794.

4. Stone GW, Ellis SG, Cox DA, et al. A polymer-based, paclitaxeleluting stent in patients with coronary artery disease. N Engl J Med 2004;350:221-231.

5. Degertekin M, Regar E, Tanabe K, et al. Evaluation of coronary remodeling after sirolimus-eluting stent implantation by serial three-dimensional intravascular ultrasound. Am J Cardiol 2003; 91:1046-1050.

6. Kim SH, Hong MK. Current status of drug-eluting stents. The Korean J Med 2008;75:370-382.

7. Morino Y, Kaneda H, Fox T, et al. Delivered dose and vascular response after beta-radiation for in-stent restenosis: retrospective dosimetry and volumetric intravascular ultrasound analysis. Circulation 2002;106:2334-2339.

8. Honda Y, Grube E, de La Fuente LM, Yock PG, Stertzer SH, Fitzgerald PJ. Novel drug-delivery stent: intravascular ultrasound observations from the first human experience with the QP2eluting polymer stent system. Circulation 2001;104:380-383.

9. Kobayashi Y, Honda Y, Christie GL, et al. Long-term vessel response to a self-expanding coronary stent: a serial volumetric intravascular ultrasound analysis from the ASSURE trial. J Am Coll Cardiol 2001;37:1329-1334.

10. Ako J, Morino Y, Honda Y, et al. Late incomplete stent apposition after sirolimus-eluting stent implantation: a serial intravascular ultrasound analysis. J Am Coll Cardiol 2005;46:1002-1005.

11. Dawkins KD, Grube E, Guagliumi G, et al. Clinical efficacy of polymer-based paclitaxel-eluting stents in the treatment of complex, long coronary artery lesions from a multicenter, randomized trial: support for the use of drug-eluting stents in contemporary clinical practice. Circulation 2005;112:3306-3313.

12. Chen JL, Gao RL, Yang YJ, et al. Comparison of short- and midterm outcomes between CYPHER and TAXUS stents in patients with complex lesions of the coronary arteries. Chin Med J 2006; 119:21-25

13. Kumar S, Suresh V, Prendergast BD, et al. Outcome in the real- world of coronary high-risk intervention with drug-eluting stents (ORCHID) -a single-center study comparing Cypher sirolimuseluting with Taxus paclitaxel-eluting stents. Catheter Cardiovasc Interv 2006;68:663-668.

14. Grube E, Dawkins KD, Guagliumi G, et al. TAXUS VI 2-year followup: randomized comparison of polymer-based paclitaxel-eluting with bare metal stents for treatment of long, complex lesions. Eur Heart J 2007;28:2578-2582.

15. Urban P, Gershlick AH, Guagliumi G, et al. Safety of coronary sirolimus-eluting stents in daily clinical practice: one-year followup of the e-Cypher registry. Circulation 2006;113:1434-1441.

16. Galløe AM, Thuesen L, Kelbaek H, et al. Comparison of paclitaxel- and sirolimus-eluting stents in everyday clinical practice: the SORT OUT II randomized trial. JAMA 2008;299: 409-416.

17. Roy P, Torguson R, Okabe T, et al. Comparison between sirolimusand paclitaxel-eluting stents in complex patient and lesions subsets. Catheter Cardiovasc Interv 2007;70:167-172.

18. Morice MC, Colombo A, Meier B, et al. Sirolimus- vs paclitaxeleluting stents in de novo coronary artery lesions. The REALITY trial: a randomized controlled trial. JAMA 2006;295:895-904.

19. Windecker S, Remondino A, Eberli FR, et al. Sirolimus-eluting and paclitaxel-eluting stents for coronary revascularization. $\mathrm{N}$ Engl J Med 2005;353:653-662.

20.Dibra A, Kastrati A, Mehilli J, et al. Paclitaxel-eluting or sirolimuseluting stents to prevent restenosis in diabetic patients. N Engl J Med 2005;353:663-670.

21. Cervinka P, Costa MA, Angiolillo DJ, et al. Head-to-head comparison between sirolimus-eluting and paclitaxel-eluting stents in patients with complex coronary artery disease: an intravascular ultrasound study. Catheter Cardiovasc Interv 2006;67:846-851.

22. Petronio AS, De Carlo M, Branchitta G, et al. Randomized comparison of sirolimus and paclitaxel drug-eluting stents for long lesions in the left anterior descending artery: an intravascular ultrasound study. J Am Coll Cardiol 2007;49:539-546.

23. Colombo A, Orlic D, Stankovic G, et al. Preliminary observations regarding angiographic pattern of restenosis after rapamycineluting stent implantation. Circulation 2003;107:2178-2180.

24. Iakovou I, Schmidt T, Ge L, et al. Angiographic patterns of restenosis after paclitaxel-eluting stent implantation. J Am Coll Cardiol 2005;45:805-806.

25. Kimura M, Mintz GS, Carlier S, et al. Outcome after acute incomplete sirolimus-eluting stent apposition as assessed by serial intravascular ultrasound. Am J Cardiol 2006;98:436-442.

26. Takeuchi H, Morino Y, Fujibayashi D, et al. Frequency and characteristics of incomplete stent apposition during and after sirolimus-eluting stent implantation. J Cardiol 2007;50:111-118.

27. Hong MK, Mintz GS, Lee CW, et al. Late stent malapposition after drug-eluting stent implantation: an intravascular ultrasound analysis with long-term follow-up. Circulation 2006;113:414-419. 